Revue d'histoire de l'Amérique française

ALS REVUE D.HISTOIRE DE L'AMÉRIQUE FRANÇAISE

\title{
Le syndicat catholique des allumettières de Hull, 1919-1924
}

\section{Michelle Lapointe}

Volume 32, numéro 4, mars 1979

URI : https://id.erudit.org/iderudit/303730ar

DOI : https://doi.org/10.7202/303730ar

Aller au sommaire du numéro

Éditeur(s)

Institut d'histoire de l'Amérique française

ISSN

0035-2357 (imprimé)

1492-1383 (numérique)

Découvrir la revue

Citer cet article

Lapointe, M. (1979). Le syndicat catholique des allumettières de Hull, 1919-1924. Revue d'histoire de l'Amérique française, 32(4), 603-628.

https://doi.org/10.7202/303730ar d'utilisation que vous pouvez consulter en ligne.

https://apropos.erudit.org/fr/usagers/politique-dutilisation/ 


\title{
LE SYNDICAT CATHOLIQUE DES ALLUMETTIÈRES DE HULL, 1919-1924
}

\author{
Michelle Lapointe \\ Québec
}

\section{Introduction}

Cette étude concernant le syndicat des allumettières de Hull veut analyser le mouvement ouvrier catholique féminin par rapport à l'ensemble des activités syndicales hulloises des années 1920. En fait, l'objet de notre recherche est de retracer l'histoire d'un syndicat qui a réussi à regrouper un nombre important de membres et à soutenir deux mouvements de grèves dans un milieu peu enclin au militantisme syndical. L'importance de ce syndicat et la combativité de ses membres consitutent donc un paradoxe intéressant à élucider.

Cette recherche a aussi un intérêt régional évident puisqu'elle situe l'action des syndiquées dans le milieu où elle s'est déroulée. En ce sens, elle reprend, dans une synthèse originale, certains éléments déjà dégagés par Brault et Maltais dans leurs recherches concernant respectivement l'histoire de Hull et celle des syndicats catholiques canadiens. Toutefois, elle nous semble avoir une perspective plus globale puisqu'elle concerne une des unions ouvrières féminines du Québec qui fonctionna le mieux à l'époque. Ainsi, l'analyse du phénomène des syndicats catholiques féminins devient possible par le biais de l'Union ouvrière des allumettières de Hull.

C'est donc dire que les deux préoccupations majeures de cet article sont, d'une part, la compréhension du militantisme de ces syndiquées, et, d'autre part, l'analyse de l'idéologie véhiculée par ce type d'organisation ouvrière. 


\section{Origine et structure du mouvement ouvrier catholique à Hull}

\section{Le syndicalisme masculin catholique à Hull}

C'est un syndicat local nommé l'Association ouvrière de Hull qui fut à l'origine du syndicalisme confessionnel dans la ville de Hull. Cette association ouvrière locale fut fondée en 1912 par M. Achille Morin avec la collaboration d'une douzaine de travailleurs de l'usine Eddy. C'est d'abord la méfiance que la compagnie Eddy avait à l'égard des syndicats internationaux qui a amené ce petit groupe à prendre une telle initiative. Une organisation ouvrière locale, croyaient-ils, obtiendrait la sympathie des employeurs. Au tout début, cette association était ouverte aux protestants comme aux catholiques. Ce n'est que vers 1915 que le syndicat devint catholique ${ }^{1}$.

En fait, cette idée de fonder une association ouvrière locale naquit, d'une part, du ressentiment des ouvriers à l'égard des unions internationales, et, d'autre part, de l'opposition de la compagnie Eddy à ces organisations ouvrières.

Cette attitude de la Compagnie se traduisit, entre autres, par de fréquents refus de négocier avec les Unions américaines. Le conflit de travail de $1903^{2}$ fut d'ailleurs l'une des occasions où la position des dirigeants de la Compagnie apparut clairement. En effet, à ce moment, l'Union des «faiseurs» de papier voulait obtenir de la Compagnie que le travail en usine s'arrête à minuit le samedi soir plutôt qu'à six heures le dimanche matin. Mais la compagnie Eddy refusa de négocier en utilisant, entre autres prétextes, l'origine de l'union ouvrière. Suite à ces mésententes, l'usine ferma pendant quelques jours. À la réouverture de celle-ci, la Compagnie refusait toujours d'entreprendre des pourparlers avec l'Union internationale. Ce refus de négocier obligea, à toute fin pratique, les ouvriers à signer une entente individuelle avec leur employeur. Les dirigeants de E.B. Eddy profitèrent de cette circonstance, et de quelques autres par la suite, pour déclarer aux ouvriers qu'ils seraient prêts à traiter avec une union locale.

Outre ces pressions de la part de la Compagnie, les ouvriers avaient accumulé, au cours des années, leurs propres griefs envers

\footnotetext{
J. Rouillard, Les Syndicats nationaux au Québec (1900-1930), Ottawa (thèse) (Université d'Ottawa, 1976), 337-340.

2 Lucien Brault, Hull 1800-1950 (Éditions de l'Université d'Ottawa, Ottawa, 1950), 154-159.
} 
leur syndicat. Celui-ci étant d'origine américaine, on l'accusait volontiers de protéger d'abord les intérêts des travailleurs américains. Que ces accusations aient découlé d'un sentiment national ou d'une situation de fait, elles n'en menèrent pas moins à la fondation d'un syndicat local, dont M. Achille Morin fut l'instigateur principal. Ce dernier avait en plus des raisons personnelles de se porter à la tête d'un tel mouvement. En effet, revenant à l'emploi de la compagnie Eddy après quelques années d'absence, il s'était vu obligé par l'Union de payer tout l'arrérage de ses cotisations syndicales.

Refusant de reconnaître la légitimité des exigences du syndicat international, Achille Morin profita de la conjoncture favorable pour fonder avec quelques amis l'Association ouvrière de Hull. Cette nouvelle association ouvrière avait comme but général la protection des travailleurs de la ville. À sa fondation, la confessionnalité n'était pas un critère d'admissibilité. Quelques mois plus tard, elle comptait déjà quelques centaines de membres.

Au début, l'Union générale regroupait des travailleurs exerçant différents métiers et elle s'appuyait sur deux structures principales: l'assemblée générale et un conseil exécutif. Les règlements généraux de l'Association étaient empruntés à la constitution des Chevaliers du Travail. Mais suite aux pressions de l'abbé Carrière, curé de St-Rédempteur, qui voyait des incompatibilités entre la doctrine sociale de l'Église et la constitution de l'Association, celle-ci en adopta une nouvelle dont les buts généraux étaient:

d'affirmer le mouvement ouvrier, de protéger et de défendre les droits de la classe ouvrière, de voir à faire aimer notre pays et à faire respecter ses lois; de réclamer pour l'ouvrier la place et le coin de terre que Dieu lui a réservés sous le soleil ${ }^{3}$.

En outre, la constitution stipulait que les membres de l'Association devaient être catholiques et ne devaient faire partie d'aucune association pouvant avoir des effets à la hausse sur le coût de la vie. Étaient donc exclus de l'Association: les banquiers, les manufacturiers, les entrepreneurs, les professionnels, les grands propriétaires terriens, les industriels, les marchands, les gérants de compagnie ainsi que tout autre groupe dont les intérêts entraient en contradiction avec ceux des ouvriers. 
Les liens d'amitié qui existaient entre le Père Guertin, supérieur et curé de la paroisse Notre-Dame de Hull, et les leaders du mouvement ouvrier, eurent aussi une certaine influence sur l'orientation du syndicat local. Discutant avec les ouvriers des expériences syndicales catholiques que connaissaient quelques pays d'Europe et la ville de Québec, il leur présenta la doctrine sociale de l'Église comme pouvant servir de tremplin à leur action syndicale.

Cette évolution du syndicat vers un engagement religieux s'est d'ailleurs faite graduellement afin de ménager les susceptibilités des protestants. Ainsi on profita de la fête du Sacré-Cœur, le 15 juin 1915, pour organiser une démonstration ouvrière qui se termina par la consécration du syndicat au Sacré-Cœur. Malgré les manifestations dirigées par le clergé catholique, quelques protestants persistaient à adhérer à l'Association ouvrière. Leur tenacité n'affecta toutefois pas la détermination manifestée par le Père Guertin dans le parachèvement de sa mission. Ainsi, il ne manquait jamais une occasion de rappeler aux ouvriers les principes de l'Union catholique. La Fête du travail fut la deuxième occasion choisie par le Père Guertin pour réorienter le syndicat. Il incita donc les ouvriers à fêter celle-ci religieusement. Une fois les ouvriers réunis, il revint à nouveau sur le sujet et il proposa de nommer un aumônier à l'Association ouvrière.

Le Père Guertin eut finalement gain de cause et réussit, à force de persévérance, à éloigner les protestants de l'association locale. Un peu plus tard, les ouvriers réunis en assemblée générale demandèrent aux autorités religieuses l'affectation d'un aumônier au service de leur syndicat. Le premier, le Père Laniel, fut nommé en 1915. Il fut remplacé par le Père Lajoie en 1918, puis par le Père Bonhomme à partir de 1919. Il va sans dire qu'à partir du moment où un aumônier participa aux activités syndicales, l'Union s'aligna étroitement sur la doctrine sociale de l'Église.

L'Association tenait des assemblée mensuelles où on discutait de l'intérêt matériel et moral des ouvriers. Un conseil exécutif, nommé par l'assemblée générale, assumait la direction du syndicat. Siégeaient à ce conseil : un chapelain, un président, deux secrétaires (archiviste et financier), deux commissaires-ordonnateurs, deux auditeurs et cinq directeurs. Aucun règlement ne fixait les cotisations des membres. Quant à leur devise, elle tenait en cinq mots d'ordre: foi, union, charité, liberté et fraternité4.

\footnotetext{
4 Ludovic Maltais, Les Syndicats catholiques canadiens (Ed. Michael J. Curley, Washington, 1925), 20.
} 
Toutefois, pendant les années qui suivirent, l'Association évolua vers une structuration professionnelle. Ainsi, l'organisation ouvrière se divisa en cinq syndicats, soit ceux des «faiseurs» d'allumettes, des policiers, des journaliers, des employés de bureau et des «faiseurs» de papier. À ces changements structuraux est venue se greffer une réorganisation administrative. L'administration syndicale fut dès lors assumée par chaque syndicat tandis que le conseil central, formé par les délégués de l'Union, veillait aux intérêts généraux des ouvriers. Ce conseil central était rattaché à la Confédération des travailleurs catholiques du Canada. À ces modifications s'ajouta aussi la disparition de l'assemblée générale et son remplacement par la convention annuelle chargée de réunir les membres afin de discuter des questions ouvrières et d'adopter de nouveaux règlements ${ }^{5}$.

Il y eut aussi, entre 1920 et 1930, la formation d'autres syndicats, au nombre de sept, se rattachant au même conseil central; tels ceux des briqueteurs-maçons, imprimeurs et relieurs, etc... Mais il ne faut pas croire que tous ces syndicats se formèrent grâce à une "prise de conscience» des travailleurs. Les représentants du clergé y jouèrent un rôle plus qu'incitateur. Ayant la main haute sur une grande partie de la presse, ainsi que sur les institutions scolaires, le clergé possédait un pouvoir de marchandage assez fort visà-vis des ouvriers, puisqu'il était lui-même employeur. À ce sujet, le Père Bonhomme raconte avec enthousiasme que lors de la formation des syndicats des plâtriers, des imprimeurs-relieurs, briqueteurs et maçons, les représentants du clergé utilisèrent leur situation d'employeur pour obtenir la syndicalisation de ces ouvriers. En fait, possédant nombre d'édifices à restaurer et à construire (écoles, églises ainsi qu'une imprimerie) ils forcèrent ni plus ni moins leurs employés à s'associer à un syndicat catholique. Pour le Père Bonhomme, ces réalisations furent des victoires du clergé contre les unions internationales ${ }^{6}$.

Toutefois, l'Église n'a pas pu faire jouer sa situation d'employeur sur les syndicats formés dans l'entreprise privée, tel celui des «faiseurs» de papier. Mais elle a utilisé toute l'influence dont elle disposait pour inciter les travailleurs à s'associer dans des syndicats catholiques. Tous ces efforts ne semblent pas avoir été très fructueux puisque sur les 3000 ouvriers catholiques que comptait la ville de

5 Ibid., 19-21.

6 R.P. Joseph Bonhomme, Notre-Dame de Hull (Archevêché d'Ottawa, Ottawa, 1931), 79-82. 
Hull en 1925, 300 seulement faisaient partie de l'Association ouvrière catholique de Hull?.

\section{Le syndicalisme féminin catholique à Hull}

Selon les sources, on situe la fondation de l'Association syndicale féminine catholique en 1918 ou en 1919. Ce syndicat fut fondé par le Père Étienne Blanchin, directeur de la Congrégation des Enfants de Marie. Il regroupait les ouvrières de la ville et peu après sa fondation, 600 membres y adhéraient. Ce syndicat féminin visait d'abord et avant tout la protection morale des jeunes filles et l'amélioration des conditions matérielles de leur travail ${ }^{8}$.

$\mathrm{Au}$ cours d'une réunion tenue en octobre $1919^{9}$, l'Association ouvrière féminine décida de se scinder en unités professionnelles. Ainsi furent fondés les syndicats des ouvrières des allumettes, du papier, de la confection et de la fourrure. Il fut convenu que chacun des syndicats enverrait des délégués au conseil central de l'association féminine ${ }^{10}$.

Ainsi, au début des années 1920, il y avait quatre syndicats de travailleuses à Hull comptant un effectif de 550 membres, alors que le nombre des ouvrières de la ville atteignait le millier ${ }^{11}$. L'organisation syndicale féminine se différenciait nettement des syndicats masculins par les buts qu'elle poursuivait. Les deux organisations n'étaient toutefois pas complètement indépendantes puisque le conseil central du syndicat masculin chapeautait l'association féminine en certains domaines.

Quant au conseil central féminin, il s'occupait principalement de l'administration des cuvres ayant pour but la formation professionnelle et l'éducation des travailleuses. Il avait la responsabilité de l'organisation de cours du soir qui étaient offerts aux ouvrières. Ces cours visaient à compléter l'éducation intellectuelle et morale des jeunes filles: cours de français, d'anglais, de sciences ménagères, de couture, etc. La contribution mensuelle qu'imposait le syndicat était de 25 sous par membre. De plus, le syndicat féminin payait une taxe au conseil central des syndicats masculins.

\footnotetext{
7 Ludovic Maltais, op. cit., 26.

8 R.P. Joseph Bonhomme, op. cit., 84.

9 Le Droit, lundi, le 6 octobre 1919.

10 La première présidente du syndicat fut Mlle Georgiane Cabana.

11 Ludovic Maltais, op. cit., 25.
} 
Le syndicat féminin était affilié au Conseil central des syndicats masculins dont il était en partie dépendant. C'est ce dernier qui avait la responsabilité de défendre les intérêts professionnels des travailleuses auprès de leurs employeurs. Le syndicat masculin s'occupait donc des conditions de travail et de salaire ainsi que des griefs des travailleuses. Il avait en plus comme fonction la promotion d'une législation protectrice du travail féminin ${ }^{12}$.

Cette situation de dépendance des syndicats féminins est nettement apparue dans les conflits de travail qu'ont vécus les employées des allumettes.

\title{
Les services aux ouvriers
}

Les services dont bénéficiaient les ouvriers, au début des années 1920 , étaient regroupés dans un édifice appelé «La Bourse de Travail», qui fut fondée par le Père Philémon Bourassa, curé de NotreDame-de-Grâce, en $1920^{13}$. On y trouvait des locaux réservés aux activités des syndicats féminins et d'autres pour celles des syndicats masculins. Ces locaux étaient utilisés pour les assemblées générales et les assemblées de comités. Quant à la section féminine, l'éditorialiste du Droit explique que:

\begin{abstract}
Elle bénéficie en plus de locaux pour les cours du soir: anglais, français, couture... Elles ont aussi une immense salle où sont donnés les cours d'art culinaire et où elles exécutent parfois des programmes de chant, de musique, voir des sauteries... entre elles seulement. C'est là qu'en un mot elles travaillent, se récréent et prient puisque toutes les réunions débutent et se terminent par la prière et la présence de l'aumônier aux assemblées ne fait qu'ajouter au côté religieux par les directions et les conseils de leur vie syndicale ${ }^{14}$.
\end{abstract}

De plus, une Caisse populaire fut fondée par l'Association pour recevoir les épargnes des ouvriers et les encourager à la pratique de l'économie. Une coopérative fut aussi établie pour eux. Celle-ci leur fournissait des biens de consommation usuels au plus bas prix possible. Il y avait aussi, à leur disposition un secrétariat permanent, qui était en fait un bureau de renseignements, ainsi qu'un bureau de

12 Ibid., 26-27.

13 Hector Legros, ptre et S œur Paul-Émile, Le diocèse d'Ottawa (Imprimerie Le Droit, Ottawa, 1949).

${ }_{14}$ Le Droit, op. cit., 24 août 1924. 
placement ${ }^{15}$. En outre, il semble qu'il y ait eu dans la plupart des syndicats catholiques, notamment ceux des femmes, des caisses de secours utilisées surtout en cas de maladie des membres ${ }^{16}$. Il nous fut impossible de savoir si de telles caisses étaient à la disposition des ouvriers de Hull. Finalement, un autre organisme fut mis sur pied par l'union ouvrière: le Cercle Benoît XV. Ouvert uniquement aux chefs syndicaux, il comportait un aspect beaucoup plus religieux. En fait, ce Cercle avait pour but d'accorder le mieux possible l'action des responsables syndicaux aux doctrines de l'Église. Il s'y tenait des réunions bi-mensuelles, des retraites fermées annuelles ainsi qu'un congrès régional qui donnait, chaque année, aux responsables et aux aumôniers syndicaux l'occasion de discuter entre eux des problèmes ouvriers ${ }^{17}$.

\section{La vie syndicale des femmes}

Les membres de l'Union ouvrière féminine de Hull furent très actives dès la mise sur pied de leur syndicat. Toutefois, les activités auxquelles participaient les unionistes semblaient être plus sociales et éducatives que syndicales. En effet, on tint à des intervalles réguliers des parties de cartes et des exercices de chant; on organisa même une soirée qui se termina par les fiançailles de leur association avec celle des hommes de la manufacture ${ }^{18}$. En dehors de ces rencontres sociales, l'initiative principale du syndicat fut l'organisation de cours du soir auxquels une bonne partie des membres s'inscrivit.

Comme on peut le constater, le syndicat féminin de manufacture de Hull était loin d'être un syndicat d'affaires, d'autant plus qu'une grande partie des revendications en ce qui concerne le traitement, les conditions de travail, etc... étaient négociées par le Conseil central du syndicat masculin. Cette union ouvrière était plutôt une forme d'encadrement social touchant tous les aspects de la vie des femmes à l'époque. Le syndicat se percevait lui-même comme une structure passagère dans la vie des femmes et comme devant contribuer à préparer la femme au rôle d'épouse et de mère qu'elle jouerait durant la majeure partie de sa vie. C'était donc une sorte d'école

\footnotetext{
15 Ludovic Maltais, op. cit., 27-28.

16 Marie Gérin-Lajoie, "Le syndicalisme féminin», in Semaines Sociales du Canada (L'Action Paroissiale, Montréal, 1922), 296.

17 R.P. Joseph Bonhomme, op. cit., 84

18 Le Droit, op. cit., septembre et octobre 1919.
} 
de la vie, de formation pratique auprès de ces futures ménagères. Bien sûr, le syndicat mettait en évidence les avantages économiques immédiats que pouvait tirer une ouvrière d'une meilleure instruction, mais les motivations avouées des cours du soir étaient de donner une formation adéquate à ces futures épouses d'ouvriers. Le commentaire de Thomas Poulin, journaliste au Droit, illustre assez bien l'idéologie des syndicats catholiques face à la femme:

L'Association catholique des jeunes filles des manufactures, vient de donner avec ses cours du soir, un exemple sur lequel nous croyons devoir quelque peu revenir, pour en souligner l'importance et indiquer un peu au moins, ce que doivent faire les jeunes ouvrières pour bien remplir leurs devoirs de futures épouses d'ouvriers...

L'organisation ouvrière féminine se distingue nettement de l'organisation ouvrière ordinaire en ce que ses membres ne doivent, d'une manière générale, n'en faire partie que pour un temps plus ou moins long, selon qu'ils entreront plus ou moins vite dans l'état du mariage.

Nous n'apprendrons rien à personne en disant que toute jeune fille qui reste dans le monde doit avoir la légitime ambition de se marier un jour. Il peut y avoir des exceptions à cette règle, mais comme l'exception est toujours le très petit nombre, nous n'insisterons pas. D'ailleurs, la majeure partie de ces exceptions n'ont décidé ou ne décident de rester vieille fille que pour continuer à élever une famille que la mère a abandonnée lorsque la mort est venue la ravir à l'amitié des siens ou pour soutenir de vieux parents... et encore là, se constituant le soutien, les jeunes filles doivent connaître l'économie et savoir la mettre en pratique.

L'économie est la base économique de tout foyer et la base de l'économie au foyer repose sur la femme. Le mari aura beau gagner de beaux salaires, s'il doit en laisser l'administration à une personne qui n'est pas économe, il en restera toujours au même point. Au contraire, il pourra se faire des revenus plutôt modiques, s'il a pour le seconder une épouse sachant travailler et connaissant la valeur de l'argent, il parviendra presque toujours à l'aisance...

Le Congrès ouvrier de Trois-Rivières a recommandé la bonne formation ménagère des futures femmes d'ouvriers... Un des moyens de bien enseigner aux futures femmes d'ouvriers à employer le plus fructueusement possible le salaire de leurs maris est certainement de leur montrer la couture. On ne sait pas assez ce que coûte de couture l'entretien d'une famille... Une femme 
qui ne sait pas coudre un bouton est certainement un désastre économique planant continuellement sur le foyer ouvrier. L'A.S.C.F. l'a compris, elle s'est empressée de répondre à la recommandation pressante du Congrès ouvrier catholique de Trois-Rivières. ${ }^{19}$

Le syndicat avait donc une responsabilité sociale à assumer. Dans ce sens, ses revendications concernant les conditions de salaire et de travail ne visaient pas qu'un but à court terme, mais elles s'inséraient plutôt dans l'optique que ces jeunes ouvrières seraient un jour des éducatrices et qu'elles devraient avoir pour ce faire, une bonne santé, un haut sens moral ainsi que les connaissances pratiques nécessaires. Toute l'organisation syndicale visait des objectifs à long terme;

Une salle pour apprendre la couture, l'art ménager, l'anglais, le français, l'arithmétique, une caisse où les ouvrières pouvaient amasser une petite dote. Donc pendant qu'au syndicat les membres se préparaient à jouer leur futur rôle de bonne ménagère ouvrière, à l'usine la présence des contre-maîtresses les protégeaient des menaces à leurs vertus qu'auraient pu constituer des contre-maîtres masculins. ${ }^{20}$

On peut donc voir que l'attitude générale envers le travail de la femme ne visait pas son élimination complète, mais avait plutôt comme but l'intégration du travail féminin à l'ensemble de la structure sociale. D'ailleurs, au début des années 1920, le nombre de femmes au travail au Canada n'était pas très élevé. On dénombrait 364831 femmes sur le marché du travail dans toute la population du pays, alors qu'au Québec elles étaient environ 93000 qui se retrouvaient surtout dans les manufactures, les magasins et les bureaux. De ce nombre, seulement $3 \%$ faisaient partie de syndicats catholiques $^{21}$. Ces chiffres nous disent jusqu'à quel point le syndicalisme catholique était un mouvement marginal à l'époque. Ce qui nous semble toutefois paradoxal dans cette question, c'est que contrairement au reste de la province et aux travailleurs de la ville de Hull, les ouvrières de cette même ville, elles, bénéficiaient d'un taux de syndicalisation de $55 \%$. Ainsi, alors qu'à Hull on faisait l'éloge de la participation des femmes à leur syndicat, ailleurs en province, on se

19 Le Droit, 29 octobre 1919.

20 L'Action Catholique, 3 décembre 1924.

21 Marie Gérin-Lajoie, «Le syndicalisme féminin» in Semaines sociales du Canada (L'Action paroissiale, Montréal 1922), 291. 
plaignait constamment du manque d'intérêt des femmes pour la syndicalisation catholique. Les justifications données à ce peu d'intérêt étaient la jeunesse, le manque de formation et d'expérience, ainsi que la timidité des femmes occupant des emplois subalternes. À ce propos, on a toutes les raisons de croire que la situation était la même à Hull, c'est-à-dire que les ouvrières étaient effectivement très jeunes, puisque ce ne fut qu'entre les années 1915 et 1920 que s'ouvrirent pour les filles, les deux dernières années du primaire. Auparavant, les institutions destinées à l'instruction des filles n'offraient que le cours primaire jusqu'à la sixième année et le cours d'École normale pour les plus nanties. Ce qui signifiait une entrée précoce dans les manufactures pour les autres ${ }^{22}$.

La faible participation des Québécoises aux activités syndicales amena toutes sortes d'organismes à se préoccuper de la question. En plus du clergé qui s'occupait abondamment de promouvoir ces associations ouvrières, d'autres institutions, telle la Fédération nationale Saint-Jean Baptiste, visaient la propagation de la syndicalisation catholique chez les ouvrières. Les «femmes d' œuvres» de la Fédération nationale se donnaient comme but de fonder et d'orienter le mouvement ouvrier féminin:

En France l'on compte sur l'action coopérative des femmes d' œuvres entièrement dévouées aux syndicats féminins et qui leur apportent le précieux concours d'une formation technique et d'un zèle apostolique reconnu.

En profitant de l'expérience acquise par nos aînées, nous ferons bien, je crois, de donner à nos syndicats le double avantage d'une contribution éclairée de la part des associées et de la part des personnes de loisir sympathiques à leurs efforts ${ }^{23}$.

Parmi ces organismes, il y avait aussi l'École sociale populaire qui, elle, offrait des services d'éducation et de direction au mouvement ouvrier féminin. Ces institutions affirmaient unanimement ne pas vouloir détruire l'initiative des intéressées; ce qu'elles voulaient surtout, c'était la subordination de ces organismes syndicaux à l'idéologie de l'Église catholique. Selon elles, il s'agissait surtout:

$1 /$ de former des ouvrières compétentes et mieux préparer à leur rôle social dans l'industrie, les professions et la famille. 2/ de maintenir en face du patronat une organisation toujours en éveil, bien que nullement agressive ${ }^{24}$.

22 R.P. Joseph Bonhomme, op. cit., 35.

23 Marie Gérin-Lajoie, op. cit., 305.

24 Ibid., 285. 
Cette coopération des «femmes d' œuvres» au mouvement syndical féminin, se basait sur l'assurance de la faiblesse de la femme en général, et de l'ouvrière en particulier, ainsi que sur le bienfait de l'Union contre un patron devant qui la femme était la plus démunie des êtres humains:

Il (syndicat) s'offre comme le moyen le plus approprié à la défense de la faiblesse native de la femme, puisque de toutes les énergies impuissantes isolément, il fait un faisceau irréversible que les plus faibles mains peuvent manier ${ }^{25}$.

La Fédération nationale Saint-Jean Baptiste se donnait pour mission, non pas de revaloriser le travail féminin, mais au moins de contribuer à le maintenir dans la dignité humaine. Ainsi, on rappelait avec émotion le Moyen âge, époque où les femmes étaient ouvrières en grand nombre et faisaient partie des corporations ouvrières au même titre que les hommes ${ }^{26}$.

À cause des liens étroits qu'elle entretenait avec le clergé, l'élite féminine de l'époque endossait à peu près complètement la position de l'Église vis-à-vis du travail de la femme. Cette attitude amenait les «femmes d'œuvres" à faire la promotion du rôle traditionnel de la femme. Toutefois, leur action ne se limitait pas à cet aspect de la question; elle visait aussi la mise sur pied de structures qui protégeraient la femme au travail. Ces "femmes d'œuvres" s'employaient donc à sauvegarder la morale, la santé des femmes qui devaient travailler. De l'avis même de Marie Gérin-Lajoie, elles essayaient en quelque sorte de limiter les dégâts. Ainsi, la meilleure façon de promouvoir le rôle traditionnel de la femme et de défendre la travailleuse contre certains abus était de circonscrire le travail de la femme à l'intérieur de cadres propres à assurer la sauvegarde de sa dignité.

\section{Les luttes ouvrières des femmes à Hull}

\section{Le premier conflit de travail}

Le premier conflit ${ }^{27}$ impliquant les «faiseuses» d'allumettes eut lieu en décembre 1919. Trois mois auparavant, à cause d'une très forte demande, la Compagnie Eddy avait proposé à ses employées

\footnotetext{
25 Ibid., 286.

26 Ibid., 288.

27 Le Droit, du samedi 13 décembre au vendredi 18, 1919.
} 
d'introduire un système de double équipe dans l'usine. Pour plusieurs d'entre elles, le travail se serait terminé à huit heures le soir. Les ouvrières avaient refusé ce nouvel horaire. D'autre part elles avaient proposé un compromis, c'est-à-dire que pendant deux mois, elles s'engageaient à travailler une journée de dix heures. Mais ce nouvel horaire ne fut pas assez productif pour la Compagnie, ce qui l'amena à proposer de nouveau le système des doubles équipes. Les allumettières rejetèrent une deuxième fois cette proposition. La Compagnie décida alors de fermer l'usine, le samedi, 13 décembre, et de négocier avec l'agent d'affaires du syndicat, Achille Morin. Mais comme les ouvrières n'étaient pas unanimes sur les ententes à signer, les négociations étaient difficiles. La Compagnie fit alors connaître aux jeunes filles son intention de réouvrir l'usine en y instaurant le roulement des équipes, une semaine de $44^{1} \frac{1}{2}$ heures et une augmentation de salaire de $25 \%$. Mais, avant de reprendre le travail, elle voulait obliger chaque employée à signer un contrat l'engageant, premièrement, à respecter ce nouvel horaire de travail tant que la Compagnie le jugerait nécessaire et l'obligeant, deuxièmement, à ne plus faire partie du syndicat. Le refus de parapher cette entente entrainait une mise à pied immédiate.

Les travailleuses n'étaient pas satisfaites de ces offres. En plus de la reconnaissance de leur syndicat, elles voulaient des garanties pour les heures et les salaires payés après la crise de production. L'usine réouvrit quelques jours plus tard, soit le mardi 16 décembre, et pendant que le travail reprenait, la Compagnie négocia avec $\mathbf{M}$. Arthur Myre, président du syndicat et M. Davis Murphy, président du syndicat des employées de la manufacture. Ceux-ci obtinrent pour les allumettières la reconnaissance du syndicat, une augmentation de salaire de $50 \%$ et l'observation de quatre fêtes religieuses par année. De leur côté, les ouvrières acceptèrent la double équipe pour trois mois ${ }^{28}$. Cette entente, bien que connue des milieux intéressés et vraisemblablement signée, ne semble pas avoir trop lié la Compagnie, puisque les employées l'accusèrent, durant les années qui suivirent, de ne pas la respecter. Durant le conflit de 1924, la Compagnie n'a d'ailleurs pas pu retrouver copie de ce contrat.

\section{Les enjeux du deuxième conflit de travail}

Le deuxième conflit ${ }^{29}$ fut beaucoup plus mémorable, sans doute parce qu'il dura plus longtemps et qu'il mobilisa beaucoup d'éner-

28 Ludovic Maltais, op. cit., 25.

29 Contre-grève à Hull (Imprimerie Le Droit, Ottawa, 1925), 1-43. 
gies. La première cause de mésentente fut l'annonce, le 2 septembre 1924, d'une baisse de salaire importante. C'était le surintendant Arthur Wood qui, aux yeux des employées, portait la responsabilité de cette décision, violant ainsi à nouveau l'entente intervenue en 1919 entre les employées et la Compagnie. Les ouvrières interprétaient ce geste de Wood comme une nouvelle provocation de sa part à leur endroit. Ce surintendant était en quelque sorte le bouc émissaire de la rancœur que les employées nourrissaient envers la Compagnie. Ainsi, après que Wood eut placardé les affiches, les ouvrières en colère abandonnèrent leur travail sur-le-champ sans même consulter la direction de leur syndicat. Les conditions qu'elles posaient pour le reprendre était l'enlèvement, par Wood lui-même, des affiches annonçant la baisse de salaire, la promesse de ne plus poser d'ultimatum de ce genre et l'amorce d'une négociation avec le syndicat comme le stipulait le contrat de 1919.

Quatre jours plus tard, soit le 6 septembre, d'autres affiches annonçaient la fermeture du département des allumettes pour une période indéterminée. Afin de justifier cette décision inattendue, la Compagnie déclara être obligée de réparer une machine de toute urgence et de disposer de surplus énormes, alors que la demande était à la baisse. Les employées croyaient toutefois que la mise à pied serait de courte durée, puisque le surintendant avait pris en note l'adresse des contremaîtresses afin de pouvoir les rappeler à l'ouvrage, avec leurs subalternes, dès l'ouverture de la manufacture. Mais bientôt, la Compagnie en profita pour déclencher une contregrève $^{30}$ en posant de nouvelles conditions de travail à ses employées à la reprise des opérations de l'usine. Le mot d'ordre des syndiquées était de ne pas accepter de nouvelles conditions de travail sans consulter le syndicat.

Le 23 septembre, la Compagnie envoie des émissaires chercher des jeunes filles pour la réouverture de l'usine. Entendant parler de ce qui se passait, les syndiquées décidèrent de se rendre à l'usine avec les jeunes filles demandées. Aux bureaux de la Compagnie, on leur présenta de nouvelles formules d'engagement, alors qu'en principe, elles étaient toutes à l'emploi de la société. Ces formules comportaient deux volets: celui d'un engagement individuel, ce qui mettait

30 Une contre-grève est en fait une traduction française de ce que nous appelons aujourd'hui un lock-out. Nous utiliserons donc indifféremment ces deux termes dans le texte. 
de côté l'ancienneté des travailleuses, et celui de la disparition de l'Union. En apprenant les dernières volontés de la Compagnie, les unionistes décidèrent d'organiser une résistance collective.

Durant tout ce conflit de travail, les ouvrières furent particulièrement excédées par la mauvaise foi de la Compagnie et par la version que donnaient les journaux anglophones du conflit. En effet, les dirigeants de l'usine ne voulaient pas admettre qu'ils avaient, à toute fin pratique, déclenché un lock-out et qu'en exigeant la signature de la nouvelle formule d'engagement, ils violaient le contrat de 1919. Ainsi, les employées de la manufacture, section des allumettes, interprétaient les gestes de la Compagnie comme voulant mettre fin à leur Union.

Les griefs des syndiquées avaient en plus une autre cible: le surintendant de leur département. Il fut l'une des figures les plus attaquées pendant tout le conflit. Les ouvrières prétendaient que par ses directives, il avait régulièrement violé le contrat de 1919 et que durant le conflit de 1924, on s'était rendu compte qu'il voulait à tout prix briser la solidarité des ouvrières ${ }^{31}$. Ainsi, il offrit à une des contremaîtresses une augmentation de salaire et un congé si elle consentait à renoncer à son Union ${ }^{32}$.

On s'en prenait bien sûr aussi aux autres dirigeants de l'usine, tel Ceasar dont on disait qu'il menait une lutte à mort contre le syndicat. Quelques-unes pensaient même que la confessionnalité du syndicat était pour quelque chose dans son hostilité. C'est ainsi qu'on interprétait ses paroles lorsqu'il déclarait: «We want to be at home». Les contre-grévistes comprenaient assez mal ce changement d'attitude des dirigeants de la Compagnie puisque, quelques années auparavant, lorsque Millen, ami du clergé, était à la tête de l'usine, les relations étaient harmonieuses entre les employées et la Compagnie. D'ailleurs les membres militants du syndicat catholique croyaient que c'était grâce à l'Union catholique que les ouvriers canadiens-français avaient atteint la valeur que louaient publiquement les patrons angloprotestants. Ainsi, les syndiquées ne comprenaient pas pourquoi ces mêmes patrons en voulaient à leur syndicat.

31 Ibid., 8.

32 Celle-ci paya son refus en étant une des dernières réengagées (20 novembre) et cela malgré les fortes pressions du syndicat. 


\section{La question morale}

Les points en litige dans ce conflit de travail ne se limitaient pas seulement à une question salariale et à la reconnaissance syndicale. S'y ajoutait une mésentente d'ordre moral. Depuis très longtemps, la surveillance immédiate des employées à la manufacture était confiée à des contremaîtresses. Comme c'était une chose normale pour les ouvrières, il n'en fut pas fait mention dans le contrat de 1919. Mais durant ce conflit, la Compagnie manifesta clairement l'intention de remplacer les contremaîtresses par une matronne et des contremaîtres, ce qui de l'avis de tous mettait en danger la vertu des jeunes filles.

Cette volonté de la société de faire disparaître les contremaîtresses répondait, selon nous, à deux impératifs: d'abord reprendre en main l'embauche et le renvoi des employées, fonctions assumées jusqu'à ce jour par les contremaîtresses, puis faire disparaître les syndiquées les plus militantes, qui étaient justement parmi les contremaîtresses. En faisant disparaître les contremaîtresses, ou tout au moins en leur enlevant le pouvoir dont elles étaient investies, la Compagnie pouvait affaiblir considérablement le syndicat, puisqu'elle pouvait engager soit des protestantes, soit des filles dont la morale serait trop douteuse pour qu'elles soient acceptées comme membres dans le syndicat catholique. Ce qui signifie qu'en réglant ce point de litige à son avantage, la Compagnie pouvait du même coup contrôler complètement le roulement de son personnel sans aucune pression externe, telles celles des contremaitresses, et ainsi affaiblir considérablement le syndicat, en engageant des travailleuses que l'Union ouvrière ne voudrait pas voir dans ses rangs.

Pour les employées, cette question semblait être strictement morale. Et pour cette raison, elle mobilisa certainement autant d'énergie que tous les autres points de désaccord mis ensemble. C'est d'ailleurs en grande partie par ce biais que le clergé et les notables de la ville s'immiscèrent dans le conflit. Évidemment, la Compagnie réagit très vivement à toutes ces pressions. $\grave{A}$ une lettre collective des curés de Hull l'enjoignant de garder les contremaitresses à son emploi pour sauvegarder la morale des jeunes filles, la compagnie répondit:

La Compagnie a toujours traité avec la plus entière considération tous les membres du clergé, mais elle ne permettra pas au clergé catholique, pas plus qu'au clergé protestant ou de toute autre dénomination, de diriger les opérations de ses usines. Ils 
ne sont pas les employés de la compagnie. Ils n'ont pas les qualifications techniques voulues et ne sont pas intéressés financièrement dans notre entreprise. ${ }^{33}$

Voyant le peu de cas que faisait la Compagnie de leurs recommandations, les curés continuèrent leurs pressions. Pour mener leurs tâches à bien, ils utilisèrent une tribune privilégiée, le prône. Et chaque fois qu'ils en eurent l'occasion, ils tentèrent de démontrer que l'employeur n'a pas plus le droit de mettre en danger la santé morale que la santé physique de ses employés:

La disparition des contre-maîtresses est plus sérieuse affaire qu' on se l'imagine. Elle fait naître un grave problème au point de vue moral. C'est ce qu'il [nous] faut prouver.

Maître de ses usines, de sa machinerie, de ses capitaux, le patron n'est pas maître de la santé, de la vertu, de la famille de ses employés. Nous ne sommes plus au temps de l'esclavage. Faire travailler ses hommes au-delà de leurs forces, exposer leur vie, compromettre leur honneur, c'est pour le patron un délit que punit même notre loi civile. Est-ce que l'âme de l'ouvrier vaut moins que son corps? La vertu d'une jeune fille a-t-elle moins de prix que ses poumons? L'hygiène a ses exigences d'air et de lumière, reconnues par la loi; la vertu elle aussi a ses exigences de sécurité et de protection, commandées par la loi divine. La promiscuité des sexes à l'atelier est déjà un grave danger moral; la dépendance immédiate de centaines de jeunes filles de quelques contre-maîtres, plus ou moins moraux, est un danger encore plus grave. Je le prouve.

Quelles que soient les vertus d'un contre-maitre, il reste toujours un homme, et comme tel n'offre pas les garanties morales que présentent des contre-maîtresses près des jeunes filles. La raison est évidente: une contre-maîtresse n'a qu'à surveiller le travail et la tenue de ses subordonnées; le contre-maître, en plus de la surveillance des subalternes, doit surveiller ses propres inclinations qui ne sont pas toujours des inclinations propres, ce n'est pas sa moindre tâche. Quelle peut bien être la défense d'une faible jeune fille contre les séductions d'un contre-maître influent et retors? Il n'y a pas de milieu: le déshonneur ou la porte. Quant on sait qu'un homme est capable de dire: «une jeune fille qui prend sa chance fait bien, j'en suis satisfait ", on devine le reste. Une femme ne peut donc raisonnablement commander à 200 hommes, et un homme ne peut commander sûrement à 200 filles. Nous prêtres nous savons à quoi nous en tenir sur cette

${ }^{33}$ Contre-grève à Hull, 11. 
question. Au reste, quand il s'agit de l'honneur de vos enfants, des futures mères de famille, il n'y a pas de risques à prendre. Supposons à la compagnie les meilleures intentions du monde, son refus de reprendre les contre-maîtresses place les ouvrières dans une situation morale inférieure à ce qu'elle était auparavant. Nous nous refusons à sanctionner cette reculade non motivée et à prendre cette responsabilité. Les pères de famille nous comprendront. ${ }^{34}$

Cette question des contremaîtresses fut ainsi débattue tout au long du conflit; elle influencera d'ailleurs son dénouement.

\section{Déroulement des négociations}

Avant que ne s'amorcent les premières négociations, les allumettières avaient commencé à piqueter devant l'usine afin d'empêcher la Compagnie d'engager d'autres ouvrières. Les dirigeants demandèrent que cesse cette activité pour entreprendre des pourparlers. Les employées refusèrent. Malgré ce refus, le 29 septembre, des échanges furent engagés entre le chapelain, les délégués du syndicat et messieurs Millen, président de la Compagnie, ainsi que Sheriff et Ceasar, respectivement vice-président et assistant-président. Pendant cette rencontre, on se mit d'accord sur sept à huit clauses d'un nouveau contrat de travail ; la paix semblait imminente. Mais le lendemain, à la reprise des négociations, le chapelain constata que l'ébauche du contrat de travail qu'on lui remit ignorait complètement les ententes intervenues la veille, puisque les clauses d'engagement individuel et de suppression des contremaitresses y apparaissaient encore. Les négociations aboutirent donc à une impasse.

Après l'échec de ces premiers pourparlers, il y eut neuf jours de silence. Puis, Bennett (ex-ministre fédéral de la justice), un des principaux actionnaires de la Compagnie, arrivant de l'Ouest, demanda de rencontrer l'aumônier. Les échanges ne donnèrent aucun résultat tangible. Finalement, le 13 octobre, un projet de contrat fut envoyé au chapelain. Dans celui-ci, la Compagnie ne s'engageait pas à reprendre toutes les contre-grévistes. Par contre, elle obligeait toutes ses employées à signer la formule d'engagement individuel. Enfin, elle enlevait aux contremaîtresses le droit d'engager et de renvoyer leurs subalternes ainsi que la chose se pratiquait depuis longtemps. Les unionistes n'étaient pas plus avancées. Elles demandèrent alors 
au gouvernement de procéder à une enquête sur le conflit. Mais la Compagnie refusa toute discussion avec les enquêteurs parce qu'elle se disait victime des contre-grévistes. En effet, les journaux du 20 octobre rapportent que, voulant franchir la ligne de piquetage, le surintendant Wood commanda à son chauffeur: «Run through the bunch». Un passant a alors sauté sur le volant de la voiture pour empêcher un accident. Le corps policier de Hull fut aussitôt mandé sur les lieux par la Compagnie afin d'assurer la protection du surintendant Wood $^{35}$. En plus d'annuler toute collaboration éventuelle de la société à l'enquête Marois, ce geste eut pour conséquence d'interrompre pendant quelques jours des négociations déjà difficiles.

Le 24, la Compagnie fit à nouveau des offres au syndicat. Ses nouvelles propositions se résumaient à la signature de la formule d'engagement et à l'embauchage des contremaîtresses sans qu'elles n'aient le droit de regard sur les engagements et les renvois des employées. Pour sa part, le syndicat refusa d'entreprendre d'autres négociations sur la base de ces offres.

Le lundi 3 novembre, des représentants de la Compagnie se rendirent chez le curé de Notre-Dame et se dirent prêts à renoncer à la formule d'engagement, à réengager les contremaitresses ainsi qu'à reprendre toutes les employées, sauf Mlle Donalda Charron, présidente du syndicat. Une fois de plus, le règlement du conflit semblait imminent. Mais lorsque le lendemain les représentants syndicaux et quelques prêtres voulurent faire signer à la Compagnie un contrat de travail contenant les promesses de la veille, celle-ci refusa en disant qu'elle ne voulait pas reprendre toutes les employées. Les prêtres proposèrent une enquête sur ces mêmes employées, mais la Compagnie refusa. Les négociations échouèrent à nouveau.

Accusée de prolonger indûment le conflit en négociant de mauvaise foi, la Compagnie voyait croître le nombre de ses détracteurs. De ce nombre, la Chambre de Commerce, les marchands de Hull et les marchands de gros d'Ottawa lui signifièrent clairement qu'ils ne voulaient plus ses produits. Même le conseil de ville, qui, le printemps précédent, avait donné à la compagnie Eddy une évaluation fixe pour dix ans, refusait maintenant à Eddy l'utilisation exclusive d'une rue. Le conseil de ville parlait même d'enlever l'accès exclusif que la Compagnie avait sur plusieurs voies de circulation. C'est

35 Le Droit, lundi, 20 octobre 1924. 
après avoir créé ce climat de tension que le conseil de ville se proposa comme médiateur; ce qui fut accepté par les deux parties.

Grâce à cette médiation, on en vint à une entente le 20 novembre. Celle-ci ne fut toutefois pas signée, mais la Compagnie s'engagea verbalement à reprendre à son emploi toutes les ouvrières et toutes les contremaîtresses congédiées en septembre. Ces dernières perdaient toutefois le droit d'engager et de renvoyer leurs subalternes. Par contre, on ne sait pas trop ce qui est advenu des baisses de salaire que la Compagnie se proposait de faire au début du conflit. Mais une chose est certaine, elle n'a pas respecté sa parole en ce qui concerne les mises à pied. En effet, entre 15 et 20 employées n'ont pas repris leur emploi après le règlement. Cette décision n'a sans doute pas empêché les opérations de l'usine, puisque par la suite, les journaux comme les autres sources d'information ne font plus mention de démêlés entre la Compagnie et ses employées de manufacture.

\section{Manifestations de solidarité}

Pendant tout le conflit, les allumettières furent très actives. En tout, elles tinrent 9 assemblées publiques afin d'expliquer leur situation aux citoyens. Les députés, les prêtres et l'élite locale prirent la parole à chacune de ces occasions. En plus de leurs protestations auprès de la Compagnie, des regroupements de citoyens, tels le Cercle des voyageurs de commerce, souscrivirent au fonds de secours des contre-grévistes. De plus, les épiciers fournirent sans aucun frais de la nourriture, et les restaurateurs des repas gratuits aux syndiquées participant au piquetage. Il y eut aussi une souscription volontaire qui permit d'amasser quelques centaines de dollars et un «tag-day», organisé par les marraines du syndicat féminin, rapporta près de $\$ 1000.00$. À cette occasion, les ouvrières parcoururent la ville en tous sens pour vendre les billets qui leur permettraient de mener jusqu'au bout la lutte qui les opposait à la compagnie Eddy. Il va sans dire que la sensibilisation de la population à ce conflit de travail eut comme conséquence immédiate le boycottage des produits Eddy. Mais, malgré l'appui massif que les Hullois accordèrent aux allumettières, il semble que les gens se fatiguèrent de la longueur du conflit. Les encouragements et les réprimandes du Droit en témoignent ${ }^{36}$.

36 Le Droit, octobre à novembre 1924. 
À notre avis, la sympathie manifestée à l'égard des jeunes filles pendant le conflit de travail est due, d'une part, à la volonté générale de voir régner un climat sain dans les usines de la ville et, d'autre part, au ressentiment que plusieurs avaient accumulé depuis des années contre la compagnie Eddy. En fait, les Hullois avaient deux séries distinctes de griefs à adresser à la société. La première série concernait surtout l'administration de l'entreprise. On reprochait à la Compagnie de ne pas ménager les mises à pied en période de récession et de continuer à payer des salaires de famine lorsque son chiffre d'affaires augmentait. La deuxième série de griefs visait le peu d'intérêt de la Compagnie à s'intégrer à la communauté de Hull et à participer à certaines activités du milieu. Ainsi, comparativement aux autres entreprises étrangères installées à Hull, la compagnie Eddy donnait bien peu aux œuvres dont les bénéficiaires se situaient dans l'Outaouais québécois. Si on ajoute à cela le fait que, dans toute l'entreprise, un seul contremaître sur huit ou neuf parlait français et qu'au bureau chef, il n'y avait aussi qu'un seul employé francophone, on peut s'expliquer le mécontentement de la population de Hull manifesté envers la Compagnie durant le conflit de 1924.

Mais l'appui aux travailleuses des allumettes n'est pas venu que de la région Hull-Ottawa. Des journaux tels L'Action Catholique et Le Devoir ainsi que des syndiqués d'autres régions ont appuyé la résistance des ouvrières ${ }^{37}$.

Quant aux quelques hommes qui travaillaient aussi à la fabrication d'allumettes, ils n'eurent pas d'intervention déterminante durant le conflit de travail. D'abord parce qu'ils n'étaient pas directement impliqués dans toutes les questions en litige et ensuite parce qu'ils étaient assez mal organisés pour prendre part au débat. En effet, ce n'est qu'après l'arrêt des opérations de l'usine que ces travailleurs décidèrent de fonder leur propre syndicat. Occupés à structurer cette nouvelle organisation, ils ne purent mettre sur pied des activités pour amasser un fonds de grève, pour sensibiliser la population au conflit de travail, etc...; ils s'en tinrent plutôt au piquetage et à la tenue de quelques réunions. Pour toutes ces raisons, les journaux donnèrent peu d'échos à leurs activités.

Il semble d'ailleurs que la section masculine ait connu la seule défection qu'il y eut parmi les syndiqués. Il s'agissait du secrétaire récemment élu de leur propre Union. Il se peut qu'il n'ait pas été

37 Contre-grève à Hull, 34-35. 
le seul «briseur de grève» (scab), mais nos sources n'en mentionnent aucun autre ${ }^{38}$.

\section{Considérations générales concernant le syndicat féminin catholique de manufacture à Hull}

Malheureusement, il ne nous a pas été possible d'avoir des renseignements substantiels sur les allumettières. Toutefois, les indices recueillis ici et là permettent de croire, qu'en général, ces ouvrières étaient de condition économique faible, célibataires et que quelquesunes d'entre elles avaient à leur charge leurs parents ou une partie de leur famille. Il semble de plus qu'elles aient été majoritairement jeunes et peu scolarisées.

Malgré ces faiblesses, elles se mobilisèrent rapidement lors des conflits de travail de 1919 et de 1924 . Il est vrai que les syndiquées avaient toujours été très militantes, ce qui explique sans doute le fait que, malgré les pressions répétées de la Compagnie, il n'y eut pas de défection pendant la contre-grève. Les appuis qu'elles ont reçus des élites locales ont probablement contribué à les maintenir fermes dans leurs exigences et solidaires dans leurs actions. Il est d'ailleurs un peu étonnant de voir l'appui inconditionnel que l'Église a donné aux contre-grévistes. Bien sûr, la question de la moralité dans l'usine y était pour quelque chose. Mais nous croyons que les motifs des représentants du clergé étaient plus complexes. En fait, pour eux, il s'agissait presque de la survie du syndicalisme catholique, dont le taux d'adhésion était assez faible chez les travailleurs de l'Outaouais. En ce sens, la bataille des jeunes filles des allumettes était stratégique puisqu'elles représentaient plus du deux tiers $(2 / 3)$ de la clientèle du syndicat catholique. Ainsi, à notre avis, c'est en grande partie pour cette raison que le clergé a appuyé les unionistes. Cette position l'a amené à ne pas ménager ses interventions au cours du conflit de travail.

Pendant toute la durée du lock-out, plusieurs des différents intervenants manifestèrent des sentiments paternalistes à l'égard des allumettières. Cette attitude les amena à marginaliser la présence des ouvrières au niveau décisionnel. Ainsi, des agents plus ou moins extérieurs au conflit prirent en main les leviers de commande au cours de la contre-grève. Par exemple, on encourageait les allumettières à

38 Ibid., 30. 
piqueter, à amasser des fonds, etc., pendant que députés, maire et prêtres, quelquefois accompagnés par la présidente du syndicat, allaient expliquer à la population les motifs du conflit de travail. Par ailleurs, toutes les négociations qui ont eu lieu pendant le lock-out se sont faites entre la Compagnie et les représentants du syndicat masculin, accompagnés de l'aumônier. La plupart du temps, les représentantes syndicales ne participaient pas aux pourparlers. De plus, c'est grâce à l'intervention du conseil de ville que s'est finalement réglé le conflit. Ce n'était pas la première fois que les édiles municipaux prenaient une telle initiative. Une intervention antérieure ayant échoué, le conseil de ville s'était engagé à ne plus se mêler de questions ouvrières. Mais il semble que la particularité de ce conflit de travail l'ait amené à intervenir de nouveau.

Certes, l'appui des élites de la ville a certainement aidé à garder le moral des troupes. Toutefois, ce qui apparaît discutable, c'est la forme qu'ont prise ces manifestations de solidarité. En fait, les ouvrières ont été, à toute fin pratique, écartées du leadership de cette lutte; lutte qu'elles avaient elles-mêmes entreprise. Car, tout au long de ces deux mois de conflit, on a vraiment l'impression que les négociations et les débats qui les concernaient directement, se faisaient par-dessus leur tête. Mais cela n'est pas vrai seulement pour la période du conflit de travail: même dans la structure syndicale, le conseil central féminin n'avait pas la responsabilité des négociations patronales-ouvrières. Ainsi, bien que ces femmes aient eu à remplir toutes les exigences du travail en usine, la structure de leur syndicat, bénéficiant de l'appui général, ne leur permettait pas d'assumer entièrement leur condition d'ouvrières. Ces femmes étaient donc marginalisées, encadrées de façon à ne pas remettre en question leur rôle social. On peut mieux comprendre pourquoi à aucun moment il ne nous a été donné de voir de dénonciations concernant le travail de la femme. C'était un phénomène trop bien circonscrit pour être menaçant. Il nous semble donc que c'est seulement dans les régions et au cours des périodes où les structures en place ont été dépassées par les événements que ce genre de propagande est apparu. Tant qu'il restait marginal, le travail des femmes dérangeait toujours un peu, mais on pouvait s'en accommoder à certaines conditions; et ces conditions, elles étaient inscrites à l'intérieur des syndicats catholiques féminins.

Dans un autre ordre d'idées, il semble assez difficile d'expliquer le fait que ce syndicat catholique féminin ait si bien fonctionné, si on compare ses effectifs à ceux des syndicats féminins du reste de la 
province et à ceux du syndicat masculin de Hull. Disposant de données trop limitées, on ne peut que faire des hypothèses à ce sujet. Ainsi, il est possible que les cours du soir offerts par le syndicat aient attiré une clientèle désireuse de compléter une formation scolaire insuffisante. D'autre part, étant donné que le mouvement syndical a émergé du groupe des Enfants de Marie, il se peut que plusieurs travailleuses aient été désireuses d'intégrer une dimension spirituelle à leur travail en usine. S'ajoute certainement à ces spéculations le fait que les ouvrières aient voulu opposer à la compagnie Eddy une organisation syndicale afin d'être mieux en mesure de défendre leurs revendications. De fait, peu de temps après la fondation du syndicat, les ouvrières étaient entrées en conflit avec la Compagnie et avaient obtenu des augmentations de salaire substantielles. Voilà, selon nous, les principales hypothèses pouvant expliquer le taux relativement élevé des adhésions à l'Association syndicale «féminine» catholique de Hull.

\section{Situation du syndicalisme catholique à Hull en 1925}

Les travailleurs de Hull avaient une faible propension au syndicalisme catholique; plusieurs raisons ont amené cet état de fait. ${ }^{39}$ En effet, les unions catholiques arrivaient dans un "marché» déjà occupé par les unions neutres. Celles-ci leur livrèrent une guerre ouverte en s'opposant à une confessionnalisation syndicale qui, selon eux, divisait les ouvriers. Cette lutte fut d'autant plus virulente que la ville d'Ottawa, où se situait le siège du Congrès des Métiers et du Travail du Canada, est sise en face de Hull. Il y avait en plus les fonctionnaires, dont plusieurs habitaient Hull, qui ne voyaient pas non plus la nécessité de cette organisation parallèle. Dès à présent, on peut voir que, dans la région, le syndicalisme catholique n'était pas très bien accueilli. Mais il y a plus, la volonté du président du syndicat catholique de participer aux élections fédérales sous la bannière du parti ouvrier en 1917, avait fortement mécontenté les deux partis officiels; cela, malgré le fait que le président en question n'ait jamais mené à terme sa volonté, puisqu'aux élections de 1917, c'est M. Joseph Eloi Lafontaine (libéral) qui fut élu par acclamation ${ }^{40}$. D'autre part, les syndiqués se divisèrent sur une campagne de

39 Ludovic Maltais, op. cit., 22-24.

40 Gouvernement du Canada, Rapport sur la treizième élection générale tenue le 17 décembre 1917 (Printer to the King most Escellent Majesty Ottawa, 1920). 
prohibition appuyée par l'association catholique. Ces malentendus entraînèrent plusieurs défections dans les rangs des unionistes catholiques. Il y eut aussi la fondation d'une coopérative alimentaire qui mécontenta les hommes d'affaires'de la région qui y virent un nouveau concurrent. Leur hostilité s'accrût encore lorsque le syndicat catholique des commis fit pression sur le conseil de ville pour obtenir la fermeture des magasins à 7 heures le samedi.

Toutes ces difficultés n'aidèrent certes pas le syndicalisme catholique à prendre de l'expansion dans la région. D'autant plus que, catholiques ou pas, un bon nombre d'ouvriers étaient indifférents aux organisations ouvrières. Cette apathie fut donc une autre raison qui, à l'époque, laissa le syndicalisme catholique dans la marginalité.

\section{Conclusion}

Partant de cette étude de cas sur le syndicalisme catholique féminin, nous croyons être en mesure de faire quelques généralisations concernant la situation de la femme des années 1920 . Selon les termes de l'époque, toute la société était unanime à concevoir la femme comme un être faible dont la vocation originale était la maternité, ce qui entraîna l'édification de structures protectrices autour des activités qu'elle exerçait hors du foyer. Ainsi, le syndicalisme catholique prévoyait un double encadrement pour la femme: d'une part, celui du syndicat féminin, et d'autre part, celui du syndicat masculin qui chapeautait l'organisme féminin. C'est donc dire qu'ayant une tâche traditionnelle à assumer, la participation de la femme à d'autres sphères d'activité étaient marginalisée, limitée et encadrée.

Dans ce contexte, le syndicat féminin a été l'une des structures qui a joué ce rôle protecteur vis-à-vis de la femme au travail. Car, si le syndicalisme catholique avait eu comme seul but la défense des intérêts des travailleurs et des travailleuses, il n'aurait pas pu justifier un dédoublement sexué des structures syndicales. D'autres impératifs sous-tendaient cette pratique. D'abord, une préoccupation d'ordre moral peu favorable à des rencontres assidues entre garçons et filles du même âge. Ensuite, la volonté d'éviter qu'en assumant la promotion de ses droits aux côtés des hommes, la femme ait pu développer une conscience de classe qui l'aurait amenée à défendre ses intérêts de travailleuse et ses intérêts de femme, se soustrayant ainsi, en tout ou en partie, aux paramètres étroits de son rôle traditionnel. En fait, on ne peut que relier la fondation des syndicats 
catholiques féminins à la volonté de conserver intact l'espace social occupé par la femme, puisque les activités des syndicats féminins se limitaient presque exclusivement à l'éducation de futures épouses et mères. Cette structure constituait en quelque sorte un cadre supplémentaire de socialisation de la femme. On ne peut expliquer autrement l'existence de cours d'art culinaire, de couture, d'arithmétique... et les divertissements sains, programmes de chant... etc. Ainsi, la volonté d'inciter la femme à réintégrer le foyer ne pouvant se faire à l'intérieur d'une union mixte, le syndicat catholique féminin devenait une nécessité. 